\title{
Fatores prognósticos relacionados à falha do tratamento não- operatório de lesões esplênicas no trauma abdominal fechado
}

\section{Prognostic factors related to non surgical treatment failure of splenic injuries in the abdominal blunt trauma}

Fábio Henrique de Carvalho, ACbC-PR'; Paula Christina Marra Romelro²; Inan Augusto Collaço, ACBC-PR³; Giorgio Alfredo Pedroso Baretta, TCBC-PR ${ }^{4}$; Alexandre Coutinho Teixeira de Freitas, TCBC-PR ${ }^{\text {; }}$ Jorge Eduardo Fouto Matias ${ }^{5}$

\section{RES U M O}

\begin{abstract}
Objetivo: Identificar fatores prognósticos relacionados com a falha do tratamento não-operatório (TNO) de lesões esplênicas no trauma abdominal fechado. Métodos: Análise prospectiva de 56 pacientes adultos submetidos à TNO e divididos em um grupo de sucesso e outro de falha, que foi definida como necessidade de laparotomia por qualquer indicação. As lesões foram diagnosticadas por tomografia computadorizada e classificadas de acordo com os critérios da AAST (American Association for Surgery of Trauma). Os parâmetros estudados foram: na admissão - pressão arterial sistólica, frequências cardíaca e respiratória, nível de consciência (Escala de Glasgow) e RTS (Revised Trauma Score); durante a hospitalização - presença de lesões associadas, transfusão sanguínea e parâmetros hematológicos, tempo de internação e ISS (Injury Severity Score). Resultados: As falhas do TNO (19,6\%) foram devidas à dor abdominal $(45,4 \%)$, instabilidade hemodinâmica $(36,4 \%)$, queda do volume globular associada a hematoma esplênico $(9,1 \%)$ e abscesso esplênico $(9,1 \%)$. Não foram observadas diferenças entre os grupos de sucesso e de falha nos dados na admissão. A taxa de falha de acordo com o grau da lesão esplênica foi $0 \%$ nos graus I e II agrupados; $17,5 \%$ nos graus III e IV agrupados e $80 \%$ no grau $V(p=0,0008)$. O uso de hemoderivados foi maior e mais frequente no grupo de falha $(p=0,05)$. As relação do ISS (Injury Severity Score) com as taxas de falha foram $0 \%$ nos pacientes com ISS $=8 ; 15,9 \%$ nos com ISS entre 9 e 25 , e $50 \%$ nos com ISS = $26(p=0,05)$. Não houve mortalidade e nem lesões de vísceras ocas despercebidas. Conclusão: O Injury Severity Score e grau da lesão esplênica relacionaram-se com a falha do tratamento não-operatório.
\end{abstract}

Descritores: Baço. Ferimentos e Lesões. Ruptura esplênica. Cirurgia.

\section{INTRODUÇÃO}

A lesões esplênicas decorrentes de trauma abdominal fechado foram tratadas quase que exclusivamente por laparotomia e esplenectomia na primeira metade do século passado. Essa conduta baseava-se nos bons resultados obtidos com a operação e na crença de que a remoção do baço não trazia consequências significativas para os pacientes, uma vez que a primeira evidência clínica favorecendo a preservação esplênica surgiu com a descrição da infecção fulminante pós-esplenectomia na década de 50 .

No final da década de 60 e início da década de 70 surge uma nova modalidade de manuseio do trauma esplênico fechado: o tratamento não-operatório (TNO). Resultados encorajadores obtidos inicialmente por cirurgiões pediátricos e posteriormente por cirurgiões gerais levaram a sua progressiva aceitação, a ponto de ser considerado atualmente o tratamento de escolha para o trauma esplênico fechado nos centros de traumatologia.
Apesar de bem sucedido na maioria dos casos, o TNO falha em algumas ocasiões e os pacientes necessitam de medidas mais agressivas e invasivas para controle. Várias tentativas têm sido feitas para identificar fatores relacionados com maior ocorrência de falha, na tentativa de refinar os critérios de seleção de pacientes, sem contudo chegar-se a consenso.

Este estudo teve como objetivo pesquisar fatores prognósticos de falha no TNO do trauma esplênico fechado em população de pacientes atendidos em pronto-socorro dedicado ao atendimento de trauma.

\section{MÉTODOS}

O estudo foi realizado no Serviço de Cirurgia Geral do Hospital do Trabalhador, vinculado à Universidade Federal do Paraná, hospital dedicado ao atendimento de pacientes traumatizados da cidade de Curitiba, região metropolitana e de cidades vizinhas, estado do Paraná, Brasil.

Trabalho realizado no Serviço de Cirurgia Geral do Hospital do Trabalhador, como parte integrante da dissertação de Mestrado do aluno Fábio Henrique de Carvalho no Programa de Pós-Graduação em Clínica Cirúrgica da Universidade Federal do Paraná.

1. Mestrando do Programa de Pós-Graduação em Clínica Cirúrgica da Universidade Federal do Paraná - UFPR-BR; 2. Aluna de Graduação do Curso de Medicina da UFPR; 3. Professor Adjunto do Departamento de Cirurgia da UFPR-BR; 4. Doutorando do Programa de Pós-Graduação em Clínica Cirúrgica da UFPR-BR; 5. Professor Adjunto do Departamento de Cirurgia da UFPR; Docente do Programa de Pós-Graduação em Clínica Cirúrgica da UFPR-BR. 
Durante um período consecutivo de 70 meses foram atendidos 137 pacientes com trauma esplênico fechado, dos quais 81 pacientes $(59,1 \%)$ foram submetidos a tratamento operatório e 56 pacientes $(40,9 \%)$ sem operação inicial. Este último grupo de pacientes constituiu a amostra de população deste estudo.

$O$ atendimento inicial às vitimas de trauma foi feito de acordo com a sistematização preconizada pelo ATLS (Advanced Trauma Life Support) do Comitê de Trauma do Colégio Americano de Cirurgiões. As vítimas de trauma abdominal fechado foram atendidas de acordo com o algoritmo apresentado na figura 1.

Os pacientes com lesões esplênicas de qualquer grau, diagnosticadas por tomografia computadorizada (TC) e estáveis hemodinamicamente eram candidatos ao TNO. A TC foi realizada em tomógrafo Siemens Somatom AR StarÒ, utilizando técnica helicoidal com cortes de $10 \mathrm{~mm}$ de espessura e reconstrução de $8 \mathrm{~mm}$. Contrastes iodados iônicos por via endovenosa, por via oral ou por sonda nasogástrica foram empregados sempre que possível. As lesões esplênicas foram classificadas de acordo com a escala padronizada pelo Organ Injury Scaling Committe da AAST (Associação Americana de Cirurgia do Trauma)'. As lesões hepáticas ${ }^{1}$ e renais ${ }^{2}$ quando presentes, também foram classificadas de acordo com as respectivas escalas, da mesma classificação.
O grau da lesão esplênica computado para análise foi o obtido com a interpretação da TC, mesmo nos casos dos pacientes submetidos posteriormente a laparotomia.

Foram incluídos os pacientes com idade igual ou superior a 14 anos, vítimas de trauma abdominal fechado, com lesão esplênica documentada por TC, cuja intenção de TNO tenha sido declarada em prontuário médico ou, na ausência desta anotação, que não tenham sido submetidos a laparotomia até duas horas pós a TC.

Foram excluídos os pacientes submetidos à laparotomia em intervalo menor que seis horas a contar da entrada no hospital ou menor que duas horas após a realização da TC de abdome inicial.

As contra-indicações absolutas foram: instabilidade hemodinâmica, sinais de peritonite ou presença de lesões abdominais de indicação cirúrgica, diagnosticadas por exames complementares. A presença de traumatismo crânio-encefálico (TCE), lesões extraabdominais com indicação cirúrgica e idade acima de 55 anos foram consideradas contra-indicações relativas. Para todos os pacientes incluídos no estudo foi aplicado o mesmo protocolo de tratamento não-operatório das lesões esplênicas, tanto durante o período de internamento hospitalar, quanto no período de acompanhamento ambulatorial (Tabela 1).

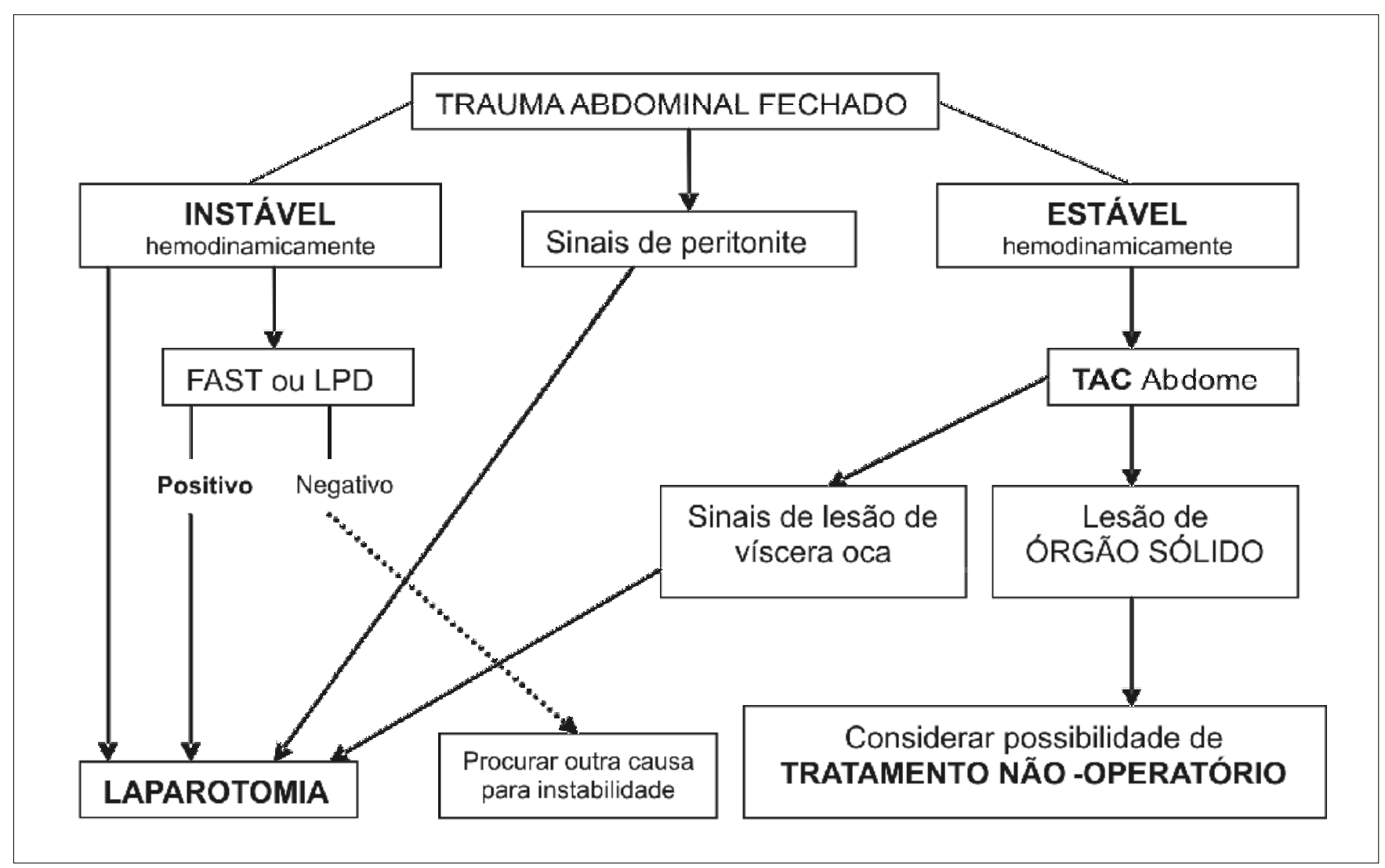

Figura 1 - Algoritmo de atendimento inicial do trauma abdominal fechado empregado no estudo*

*Modificado de: American College of Surgeons, Committee on Trauma. Advanced Trauma Life Support (ATLS). Instructor Course Manual. 6. ed. Chicago: American College of Surgeons, 1997. 
Tabela 1 - Protocolo de tratamento não-operatório das lesões esplênicas no trauma abdominal fechado.

\author{
Internação \\ - Observação no PS ou UTI por 72 horas. \\ Exame clínico seriado com controle de dados vitais a cada \\ $2 \mathrm{~h}$ por 12 horas e a cada $4 \mathrm{~h}$ por 24 horas. \\ Hemograma na entrada e 6, 12, 24 e 48 horas. \\ Amilase diária até o terceiro dia. \\ - Repouso absoluto por 48 horas ou até estabilização do VG. \\ - Jejum até re-estabelecimento do trânsito intestinal (média 24 h). \\ - TAC de controle no sétimo dia. \\ - Alta no sétimo dia de internação.
}

\section{Ambulatório}

Retorno em sete dias para avaliação clínica.

- US de controle em 30 dias.

Outros exames de imagem e laboratoriais a critério clínico Repouso relativo domiciliar por no mínimo 30 dias, estendido a critério clínico de acordo com o grau da lesão e atividades habituais do paciente.

\section{Parâmetros do estudo}

As seguintes variáveis de estudo foram coletadas e tabuladas, com auxílio de planilha eletrônica, para análise de seu poder em predizer falha do TNO:

a) Da entrada no pronto socorro: pressão arterial sistólica em mm Hg; frequência cardíaca em batimentos por minuto; frequência respiratória em movimentos respiratórios por minuto; nível de consciência de acordo com a Escala de Coma de Glasgow (GCS) ${ }^{3}$; Revised Trauma Score $^{4}=0,9368$ GCS $_{p}+0,7326$ PAS $_{p}+0,2908$ FR $_{p}$ onde: $\mathrm{GCS}=$ Escala de coma de Glasgow; PAS = Pressão arterial sistólica; FR = Frequência respiratória (os valores unitários de cada parâmetro são divididos em cinco categorias diferentes, com valores atribuídos de 4 a zero de acordo com desvio da normalidade.

b) Da internação e ambulatório: presença de lesões associadas, classificadas de acordo com o Abreviatted Injury Score (AIS) ${ }^{5}$; quantidade de transfusão sanguínea em unidades de concentrado de hemácias; valores iniciais de entrada, e os menores valores durante o internamento, da hemoglobina $(\mathrm{Hb})$ em $\mathrm{g} / \mathrm{dL}$ e volume globular (VG) em \%. No caso dos pacientes com falha do TNO foi computado o menor valor da Hb e VG antes da realização da laparotomia; tempo de internação em dias; Injury Severity Score ISS ${ }^{5}=$ $\mathrm{AIS}_{(1)}^{2}+\mathrm{AIS}_{(2)}^{2}+\mathrm{AIS}_{(3)}^{2}$ onde: AIS = Abreviatted Injury Score (análise de severidade do trauma das três lesões mais severas, presentes em regiões corporais distintas, graduadas de 1 a 6 de acordo com tabelas específicas).

\section{operatório}

Definição de falha do tratamento não-

A falha foi definida como a necessidade de interrupção do TNO para a realização de laparotomia, por qualquer indicação. Foram avaliados o tempo de ocorrência da falha, a causa da falha e os achados na laparotomia. Os pacientes foram subdivididos em grupos de sucesso e de falha, de acordo com o resultado do TNO.

\section{Análise estatística}

Utilizou-se para os cálculos o programa estatístico STATISTICA ${ }^{\circledR}$ versão 6.0 (2001) da StatSoft, Inc. As variáveis numéricas foram descritas como média \pm desvio padrão (DP). As diferenças entre variáveis numéricas foram analisadas pelo teste t de Student ou pelo teste Mann-
Whitney, quando as condições de normalidade e homogenicidade de variâncias não fossem obedecidas. As diferenças entre proporções das variáveis categóricas foram analisadas com o teste Qui-quadrado e com o teste exato de Fisher, quando houvesse frequências esperadas abaixo de cinco em tabelas $2 \times 2$. Determinou-se o nível de significância em $5 \%(p=0,05)$.

\section{RESULTADOS}

Os acidentes de trânsito foram a causa mais frequente das lesões esplênicas, com 39 casos $(69,6 \%)$, seguidos pelas quedas, com 11 casos (19,6\%) e agressões, com três casos $(5,4 \%)$. Não houve mortalidade na presente série.

A distribuição dos pacientes de acordo com o grau da lesão esplênica encontra-se na figura 2. Nota-se distribuição normal dos pacientes entre os cinco diferentes graus de lesão esplênica.

A idade no grupo de falha variou de 17 a 65 anos, com média de 33,3 $\pm 17,8$ anos, e no grupo de sucesso variou de 14 a 50 anos, com média de 26,9 \pm 9,4 anos $(p=0,4516)$. Dois pacientes $(3,6 \%)$ com idade superior a 55 anos apresentaram falha no TNO por instabilidade hemodinâmica. A taxa de falha para o grupo de pacientes abaixo de 55 anos foi de 16,7\%. Não houve diferença entre os sexos com relação à ocorrência de falha com $20 \%$ para o sexo feminino e $19,6 \%$ para o masculino $(p=0,6802)$.

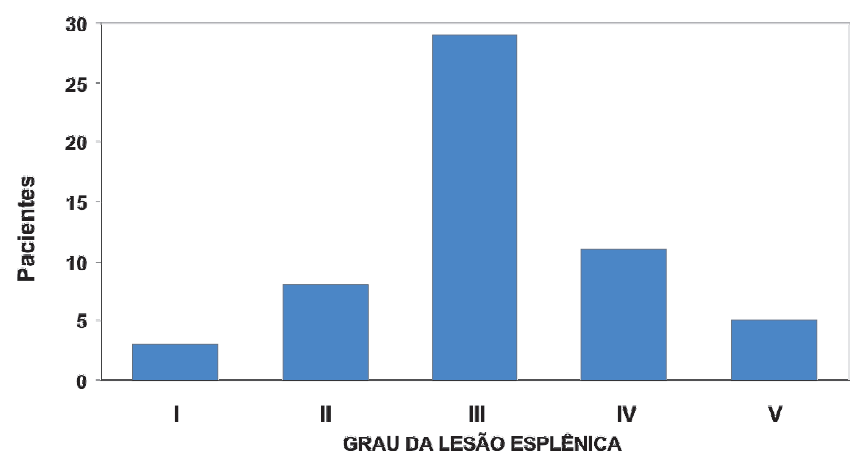

Figura 2 - Grau de lesão esplênica dos pacientes submetidos a tratamento não-operatório. 


\section{Causas e tempo das falhas}

O TNO foi bem sucedido em 45 pacientes $(80,4 \%)$ e falhou em 11 (19,6\%).

As causas de falha no TNO estão relacionadas na tabela 2. O tempo de ocorrência das falhas obedeceu distribuição bimodal, com nove casos $(81,8 \%)$ ocorrendo entre 8 e 79 horas, com média de $24,4 \pm 21,9$ h, e os restantes dois casos $(18,1 \%)$ ocorrendo no $13^{\circ}$ e $14^{\circ}$ dias de tratamento; $54,5 \%$ das falhas ocorreram antes de 24 horas.

\section{Dados da avaliação inicial, grau de lesão esplênica e lesões associadas \\ Os valores médios da PAS, FC, FR e RTS nos gru-} pos de sucesso e falha do TNO encontram-se na tabela 3. Não houve diferenças significativas nos valores dos parâmetros da avaliação inicial entre os grupos de falha e sucesso no TNO.

O grau médio da lesão avaliado por TC e classificado de acordo com a AAST ${ }^{1}$ foi de 2,9 $\pm 0,9$ no grupo de sucesso e de $3,9 \pm 0,9$ no grupo de falha $(p=0,01)$. A taxa de falha do TNO foi de $0 \%$ nos pacientes com lesão grau I e II, $17,2 \%$ nos com grau III, $18,2 \%$ nos com grau IV e $80 \%$ nos com grau V. Detectou-se correlação direta entre o grau de lesão esplênica e a ocorrência de falha no TNO.

A distribuição das lesões associadas com AIS = 2, de acordo com sua frequência nos grupos de sucesso e falha, encontra-se na tabela 4 . Não houve diferença entre a incidência de lesões associadas $(62,2 \% \times 45,4 \% p=$ $0,2494)$ e média de lesões por paciente $(2 \pm 1,0 \times 1,4 \pm$ $0,9 p=0,2004)$ entre os grupos de sucesso e de falha, respectivamente. Lesões de fígado ou rim associadas ocorreram em 17 de 45 pacientes do grupo de sucesso $(37,8 \%)$

Tabela 2 - Causas de falha do tratamento não-operatório.

\begin{tabular}{lrc}
\hline Causas & \multicolumn{2}{c}{ Pacientes } \\
\cline { 2 - 3 } & $\mathrm{n}$ & $\%$ \\
\hline Dor abdominal & 5 & 45,4 \\
Instabilidade hemodinâmica & 4 & 36,4 \\
Aumento do hematoma e queda do VG & 1 & 9,1 \\
Abscesso esplênico & 1 & 9,1 \\
Total & 11 & 100 \\
\hline
\end{tabular}

Tabela 3 - Dados da avaliação inicial nos grupos de sucesso e falha do tratamento não-operatório.

\begin{tabular}{lcccc}
\hline Dado & $\begin{array}{c}\text { Sucesso } \\
\text { (média } \pm \text { DP) }\end{array}$ & $\begin{array}{c}\text { Falha } \\
\text { (média } \pm \text { DP) }\end{array}$ & $p$ \\
\hline PAS & $126 \pm 18,3$ & $126 \quad \pm 25$ & $0,9298^{(1)}$ \\
FC & $88,5 \pm 16,3$ & $89 \pm 20,2$ & $0,9326^{(1)}$ \\
FR & $21,8 \pm 3,7$ & $23,2 \pm 4,7$ & $0,2610^{(2)}$ \\
RTS & $7,83 \pm 0,06$ & $7,81 \pm 0,09$ & $0,8125^{(2)}$ \\
\hline
\end{tabular}

(1) Teste $t$ de Student (2) Teste $U$ de Mann-Whitney.

$P A S=$ pressão arterial sistólica; $F C=$ frequência cardíaca; $F R=$ frequência respiratória; RTS = Revised Trauma Score. e em nenhum paciente do grupo de falha ( $p=0,0113)$. Nenhum paciente apresentava lesão simultânea dos três órgãos (fígado, rim e baço).

No grupo de sucesso, o ISS variou de 5 a 34 , com média de 15,2 $\pm 7,1$ e no grupo de falha, variou de 10 a 38, com média de $18,7 \pm 9,4$ ( $p=0,2198)$. Quatro pacientes apresentaram ISS $=8$, 44 ISS entre 9 e 25 e oito ISS $=26$. Detectou-se correlação direta entre o ISS e a ocorrência de falha no TNO.

Dois dos 11 pacientes operados (18,2\%) apresentavam lesões intra-abdominais associadas. Um com hematoma de cólon transverso e outro com contusão da cauda do pâncreas (ambas lesões grau I pela classificação AAST). Nenhum paciente apresentou perfuração de víscera oca abdominal.

Exames laboratoriais, transfusão sanguínea e tempo de internação

A tabela 5 mostra os valores de volume globular e hemoglobina detectados na admissão e seus menores valores durante a internação nos dois subgrupos estudados. Não houve diferença estatística entre os subgrupos em nenhum dos parâmetros analisados.

Tabela 4 - Lesões associadas com AIS = 2 nos grupos de sucesso e falha do tratamento não-operatório.

\begin{tabular}{lcccc}
\hline Lesão & $\begin{array}{c}\text { Sucesso } \\
(\mathbf{n})\end{array}$ & $\begin{array}{c}\text { Falha } \\
(\mathbf{n})\end{array}$ & \multicolumn{2}{c}{$\begin{array}{c}\text { Total } \mathbf{n} \\
\text { (\% }\end{array}$} \\
& 12 & - & 12 & dotal) \\
\hline Lesão renal & 9 & 2 & 11 & $(19,0)$ \\
Fratura de arcos costais & 6 & - & 6 & $(9,5)$ \\
Hemopneumotórax & 5 & - & 5 & $(7,9)$ \\
Lesão hepática & 3 & 1 & 4 & $(6,3)$ \\
TCE leve & 3 & - & 3 & $(4,8)$ \\
Fratura de fêmur & 1 & 1 & 2 & $(3,2)$ \\
Fratura de escápula & 2 & - & 2 & $(3,2)$ \\
Fratura de pelve & 2 & - & 2 & $(3,2)$ \\
Fratura de tornozelo & 2 & - & 2 & $(3,2)$ \\
Fratura de ulna & 2 & - & 2 & $(3,2)$ \\
Fratura de úmero & 9 & 3 & 12 & $(19,0)$ \\
Outras lesões & 56 & 7 & 63 & $(100)$ \\
Total & &
\end{tabular}

Nota: dado igual a zero. AIS = Abreviatted Injury Score.

Tabela 5 - Volume globular (VG) e hemoglobina (HB) na admissão e os menores valores durante a internação nos grupos de sucesso e de falha no tratamento não-operatório.

\begin{tabular}{lccc}
\hline Dado & $\begin{array}{c}\text { Sucesso } \\
\text { (média } \pm \text { DP) }\end{array}$ & $\begin{array}{c}\text { Falha } \\
\text { (média } \pm \text { DP) }\end{array}$ & $\mathrm{p}^{(1)}$ \\
\hline VG na entrada & $35,6 \pm 6,8$ & $35,2 \pm 3,9$ & 0,8581 \\
Menor VG & $29,5 \pm 6,3$ & $30,4 \pm 6,1$ & 0,5788 \\
Hb na entrada & $11,9 \pm 2,2$ & $11,5 \pm 1,4$ & 0,5033 \\
Menor Hb & $9,9 \pm 2,2$ & $10,1 \pm 2,1$ & 0,8484 \\
\hline
\end{tabular}

(1) Teste $t$ de Student. 
Dos 56 pacientes, $21(37,5 \%)$ receberam transfusão de concentrado de hemácias, 14 (31,1\%) no grupo de sucesso e sete $(63,6 \%)$ no grupo de falha $(p=0,0511)$. O número de unidades transfundidas por paciente foi de $0,9 \pm 1,9$, no grupo de sucesso e de $2,1 \pm 1,9$, no grupo de falha $(p=0,0488)$. Quando comparou-se o mesmo parâmetro entre apenas aqueles que receberam hemoderivados, o número de unidades transfundidas por paciente foi de 3,1 $\pm 2,3 \cup$ no grupo de sucesso e de 3,3 \pm $1,1 \cup$ no grupo de falha $(p=0,2631)$.

O tempo de internação variou de quatro a 16 dias (média de $8,8 \pm 2,9$ ) no grupo de sucesso do TNO. No grupo de falha variou de quatro a 29 dias, com média de $10,8 \pm 8,2(p=0,8045)$.

\section{DISCUSSÃO}

O TNO das lesões esplênicas em trauma abdominal fechado obtém sucesso na maioria das séries atuais publicadas, com taxas de falha que variam de $2 \%$ a $33 \% 6,7$.

As causas para interrupção do TNO são múltiplas na literatura e em algumas séries não chegam a ser especificadas $^{8}$. A suspeita de lesões de vísceras ocas associadas é a principal razão da indicação da laparotomia por piora da dor abdominal. Em décadas passadas, as incidências relatadas de $26,5 \%$ de lesões de vísceras ocas em pacientes adultos com trauma abdominal fechado ${ }^{9}$ e de $37 \%$ de lesões intra-abdominais associadas em pacientes com lesões esplênicas ${ }^{10}$ sugeriam elevado risco de ocorrência de lesões despercebidas em pacientes que fossem submetidos a TNO. No entanto, estudos posteriores relataram ausência de lesões despercebidas ${ }^{11,12}$ ou mesmo índices muito baixos, por volta de $1 \%{ }^{13,14}$, provavelmente efeito de adequada seleção de pacientes, com exclusão daqueles com sinais de peritonite na admissão ou com piora precoce da dor abdominal. Nesta série, a piora da dor abdominal foi a indicação mais frequente para laparotomia. Entretanto, nenhuma perfuração de víscera oca foi encontrada nos pacientes operados e apenas um apresentava contusão pancreática que poderia ser responsabilizada pela piora da dor. Esse resultado reflete a ansiedade gerada pela possibilidade de lesões despercebidas ${ }^{6}$ e ocorreu também em outras séries ${ }^{15}$.

Instabilidade hemodinâmica foi a segunda causa mais frequente de interrupção do TNO. Enquanto algumas séries não apresentaram falhas por instabilidade ${ }^{16,17}$, em outras ela foi responsável por $100 \%$ das falhas ${ }^{12,18}$. Essa variação pode ser explicada por possíveis diferenças entre as populações de pacientes das séries e também por diferentes definições de instabilidade hemodinâmica. O julgamento médico sobre a estabilidade hemodinâmica varia entre diferentes hospitais e entre médicos de um mesmo hospital ${ }^{13}$ e mesmo serviços que utilizaram critérios objetivos para definir a instabilidade em seus protocolos, deixaram a cargo do cirurgião a classificação final do paciente ${ }^{19}$. A interpretação da literatura é ainda dificultada pela utilização do termo "hemorragia" como causa de falha, en- globando tanto pacientes com instabilidade quanto com queda progressiva do VG ${ }^{6,11}$.

Um paciente desta série foi operado por queda do VG, associada a aumento de hematoma subcapsular, verificado pela TC. A queda do VG foi a maior responsável pela interrupção do TNO em várias séries ${ }^{13,20,21}$ e as principais justificativas para laparotomia são a possibilidade de sangramento lento ou de repetição, sem repercussão hemodinâmica, com riscos da hemotransfusão maiores que os benefícios do TNO. Não foi considerado isoladamente a queda do VG como indicação para laparotomia, por ser dependente de lesões associadas e procedimentos cirúrgicos, principalmente ortopédicos.

A indicação de laparotomia por alterações no aspecto da lesão esplênica ou aumento do volume de hemoperitônio na TC foi relatada por vários autores ${ }^{16,19,20,22}$. No entanto, a repetição da TC como rotina não alterou o tratamento dos pacientes em alguns estudos, sugerindo que sua repetição estaria indicada apenas em pacientes com lesões mais severas ou com alguma indicação clínica ${ }^{23,24}$. Enquete realizada entre os membros da EAST (Eastern Association for the Surgery of Trauma) mostrou que, embora a maioria não utilize $\mathrm{TC}$ de rotina para reavaliação das lesões esplênicas, $85 \%$ deles a repetem em alguns subgrupos de pacientes, como os com lesões de grau maior ou com achado de extravasamento de contraste no exame inicial ${ }^{25}$.

$\mathrm{Na}$ literatura os resultados do TNO em pacientes acima de 55 anos de idade - divisor amplamente aceito e utilizado - apresentam ampla variação 12,21,22,26,27. Entretanto, para os mesmos graus de lesão esplênica e lesões associadas parece haver maior taxa de falha em pacientes acima de 55 anos. Nesta casuística, o número reduzido de pacientes acima dessa idade não permite conclusões seguras.

Utilizou-se neste estudo, na admissão do paciente, somente dados objetivos mensuráveis ou calculados. PAS e a FC iniciais não discriminaram pacientes com sucesso ou falha no TNO, achado muito comum na literatu$\mathrm{ra}^{13,20}$. Este dado sugere que os dados vitais iniciais são úteis para a definição do estado hemodinâmico e portanto seleção dos pacientes para o TNO, mas não para previsão do desfecho do tratamento.

As alterações decorrentes de TCE ou intoxicações, por dificultarem ou impossibilitarem o exame físico abdominal confiável do paciente, considerado fundamental para detecção de lesões de vísceras ocas associadas que não tivessem sido detectadas pelo exame físico inicial ou pela TAC eram consideradas critérios de exclusão para TNO. Entretanto, séries publicadas excluindo pacientes com TCE, apresentaram baixa incidência de lesões de vísceras ocas associadas, sugerindo que o TNO poderia ser tentado em pacientes com alterações do nível de consciência ${ }^{13-15,24,28}$.

A correlação entre resultado do TNO e severidade da lesão esplênica só foi possível após o surgimento da TAC, método que possibilita melhor delineação da lesão que a arteriografia, a cintilografia e a ultrassonografia. A gradação de lesão esplênica pela classificação da AAST não é método exato e está sujeito à variações significati- 
vas. O grau da lesão esplênica avaliado pela TC pode sofrer discordâncias entre observadores e em ocasiões diferentes, pelo mesmo observador. Apesar dessas limitações, a classificação da AAST permanece como a melhor para uso no trauma esplênico e foi empregada nesse estudo para avaliar a severidade morfológica das lesões esplênicas.

Há resultados consistentes na literatura relatando relação direta entre taxa de falha e grau da lesão esplênica ${ }^{13,15,19,22,28}$. Lesões grau I e II têm taxas de falha uniformemente baixas ${ }^{13,14,28}$. Já pacientes com lesões grau III e IV apresentam ampla variação na literatura ${ }^{15,28}$. Uma das possíveis razões para as variações observadas é o fato da interpretação da TC ser dependente do observador, principalmente na diferenciação entre lesões grau III e IV, o que pode ocasionar diferenças significativas na classificação das lesões entre instituições. Esse fator pode ter sido responsável pela observação de taxas de falha equivalentes nas lesões grau III e IV neste estudo, enquanto que a literatura em geral reporta taxas de falha maiores para as lesões grau IV, que para as de grau III13,15,22,28. Não obstante, a maioria dos pacientes com lesões grau IV (até 75\%) apresenta graus variáveis de instabilidade hemodinâmica na entrada no hospital e é submetida ao tratamento operatório, ao contrário dos pacientes com lesões grau III, que são na maioria dos casos submetidos ao TNO ${ }^{13,15,19}$.

Os pacientes com lesões grau $V$ são submetidos ao tratamento operatório em cerca de $90 \%$ dos $\operatorname{casos}^{13,19}$. As taxas de falha reportadas são em geral superiores a $75 \% 13,15,22$, fato também observado no presente estudo.

O volume do hemoperitônio não pôde ser incluído como parâmetro deste estudo. A sua quantificação exige TC de abdome e pelve e apenas $43 \%$ dos pacientes do estudo realizaram TC de pelve, dado que motivou alteração no protocolo de TNO do hospital, com a inclusão da TC de pelve para todo paciente que tenha lesão detectada em TC de abdome superior.

Na casuística desse estudo, não foi detectado extravasamento de contraste. A baixa incidência pode ser resultante da técnica de infusão manual de contraste empregada no serviço: idealmente para detecção de extravasamento, a infusão deve ser feita por bomba com fluxo mínimo de $2 \mathrm{~mL} / \mathrm{s}^{11}$.

Lesões associadas foram frequentes na casuística analisada e sua incidência não divergiu nos grupos de sucesso e de falha do TNO, concordando com as observações de outros autores 6 .

Lesões hepáticas e renais estão frequentemente associadas às lesões esplênicas ${ }^{21}$, não havendo contra-indicação para o TNO e esperando-se taxas de falha semelhantes às dos pacientes com lesões esplênicas isoladas.

O ISS médio dos pacientes dessa casuística está de acordo com o a prevalência na literatura, em torno de $15^{22}$. Alguns autores ${ }^{6,13,22}$ observaram que pacientes com falha no TNO apresentavam ISS mais elevado, enquanto que outros ${ }^{20,21}$ não observaram tal diferença. Nesta casuística não foi encontrada diferença significativa entre o ISS médio do grupo de falha e de sucesso do TNO. Contudo, quando estratificados por faixa de ISS, à semelhança do estudo de Mclntyre et al. ${ }^{8}$, houve diferença na taxa de falha: paci- entes com maior ISS apresentaram maior taxa de falha do TNO.

Na literatura, a associação de valores maiores do ISS com a taxa de falha é atribuída à gravidade do trauma decorrente de múltiplas lesões. No entanto, como o grau da lesão esplênica tem peso no cálculo do ISS, não se pode afirmar que o ISS tenha influência independente na taxa de falha. Como modo de evitar essa influência foi calculado também o ISS dos pacientes excluindo-se a lesão esplênica, e foi observado que os valores obtidos não apresentaram diferença significativa, não permitindo portanto relacionar a presença de lesões associadas com a taxa de falha.

Não foi observada diferença nos valores do VG e da HB de entrada entre os grupos de falha e de sucesso do TNO, em congruência com outras séries ${ }^{20}$. Os menores valores de VG e HB durante todo o internamento ou até a indicação de laparotomia não forma discriminatórios entre pacientes com e sem falha do TNO. Os valores do VG e HB podem ser utilizados para monitorar a continuidade do sangramento apenas até determinados limites, a partir dos quais emprega-se a transfusão sanguínea. O número de unidades transfundidas torna-se então melhor indicador da persistência ou repetição do sangramento, dado importante na decisão de manter ou interromper o TNO.

Alguns protocolos publicados recomendavam a interrupção do TNO se necessária transfusão de mais de quatro unidades de hemoderivados, que não justificada por outras lesões ${ }^{12,14}$. No protocolo adotado neste estudo não foi definido limite máximo de unidades aceitável para pacientes em TNO, devendo cada situação ser avaliada individualmente. A proporção de pacientes que necessitou de transfusão e o número de unidades transfundidas por paciente foram superiores no grupo de falha em relação ao grupo em TNO. Este achado repete o observado em outros $\operatorname{artigos}^{13,14,20}$. Considerando-se a necessidade de transfusão nos pacientes com falha do TNO inferior ${ }^{13}$ ou equivalente ${ }^{14}$ à dos pacientes submetidos ao tratamento operatório inicial, poder-se-ia argumentar que a tentativa de TNO não exporia esses pacientes a necessidade maior de transfusão do que aquela que necessitariam se inicialmente operados. Esta afirmativa não pode ser comprovada na base na literatura ou no estudo em análise, visto que os grupos de pacientes submetidos ao TNO e ao tratamento operatório inicial são dispares.

Cerca de um terço dos pacientes submetidos ao TNO com sucesso recebeu hemoderivados, proporção semelhante à observada em outros estudos ${ }^{13,18}$. O número médio de 0,9 U de concentrado de hemácias por paciente do grupo de sucesso do TNO, embora superior a valores em torno de 0,5 U reportados por alguns autores ${ }^{6,22}$, foi consideravelmente inferior ao valor de $3 \mathrm{U}$ observado em algumas séries ${ }^{14,20,21}$. O número médio de 2,1 U de concentrado de hemácias recebidos por paciente do grupo de faIha do TNO foi comparável com o menor valor relatado na literatura, de 2,4 U22; e inferior aos valores acima de $3 \mathrm{U}$ geralmente relatados ${ }^{13,20,21}$.

Considerando-se somente os pacientes que foram transfundidos, não houve diferença entre o volume de 
hemoderivados recebidos pelos pacientes do grupo de faIha e de sucesso do TNO. Estes resultados sugerem que o TNO não associou-se a utilização excessiva de transfusão de hemoderivados, tanto nos casos de sucesso quanto de falha do tratamento.

Existe mortalidade em pacientes vítima de trauma grave. Esse fato não difere em pacientes em TNO. Semelhante a este estudo, Pachter et al. ${ }^{29}$ não observaram mortalidade em suas séries, possivelmente devido à ausência de pacientes com lesões associadas severas, principalmente TCE, nas casuísticas. A avaliação dos registros médicos de pacientes que foram à óbito em série multicêntrica encontrou falhas na seleção dos pacientes para o TNO e na interpretação de exames diagnósticos, que relacionavamse diretamente com a mortalidade em $40 \%$ dos $\operatorname{casos}^{30}$.
Recomenda-se emprego de protocolos formais e julgamento criterioso na seleção de pacientes e na condução do TNO. O tratamento operatório de lesões esplênicas e o abandono a tempo do TNO continuam terapêuticas adequadas a determinados grupos de pacientes e não são razões de demérito para um cirurgião ou serviço.

No presente estudo não foi observada relação entre a falha do tratamento não-operatório do trauma esplênico fechado com a idade, sexo, dados vitais iniciais, valores do volume globular e hemoglobina e presença de lesões associadas.

O ISS e o grau da lesão esplênica apresentaram relação direta e significativa com a taxa falha do tratamento não-operatório.

\title{
A B S T R A C T
}

\begin{abstract}
Objective: Identify prognostic factors related to treatment failure of blunt splenic injuries managed by non surgical treatment (NST). Methods: Fifty six adult patients submitted to NST were prospectively studied. The injuries were diagnosed by computed axial tomography scan and classified according to AAST (American Association for Surgery of Trauma) criteria. Patients were divided in success and failure groups. NST failure was defined as the need for laparotomy for any reason. Results: NST failures (19.6\%) were due to: abdominal pain (45.4\%), hemodinamic instability (36.4\%), splenic haematoma associated to a fall in hematocrit (9.1\%) and splenic abscess $(9.1 \%)$. There were no failures in grade I and II of the splenic injuries; failure rate was $17.5 \%$ in grade III and IV injuries grouped, and $80 \%$ in grade $V$ injuries $(p=0,0008)$. In the success group, 31.3\% patients received red cell transfusions, versus $63.6 \%$ patients in the failure group $(p=0,05)$. Failure rate in patients with ISS = 8 was zero; $15.9 \%$ in patients with ISS 9 to 25 ; and $50 \%$ in patients with ISS $=26(p=0,05)$. There were no deaths or missed bowel injuries. Conclusion: ISS and splenic injury grade were related to failure of NST.
\end{abstract}

Key words: Spleen. Wounds and injuries. Splenic rupture. Surgery.

\section{REFERENCIAS}

1. Moore EE, Cogbill TH, Jurkovich GJ, Shackford SR, Malangoni MA, Champion HR. Organ Injury scaling: spleen and liver (1994 Revision). J Trauma. 1995; 38(3):323-4.

2. Moore EE, Shackford SR, Pachter HL McAninch JW, Browner BD, Champion HR et al. Organ injury scaling: spleen, liver and kidney. J Trauma. 1989; 29(12):1664-6.

3. Teasdale G, Jennett B. Assessment of coma and impaired consciousness. A pratical scale. Lancet. 1974; 2(7872):81-4.

4. Champion HR, Sacco WS, Copes WS, Gann DS, Gennarelli TA, Flanagan ME. A revision of the trauma score. J Trauma. 1989; 29(5):623-9.

5. Baker SP, O'Neill B, Haddon W Jr, Long WB. The injury severity score: a method for describing patients with multiple injuries and evaluating emergency care. J Trauma. 1974; 14(3):187-96.

6. Velmahos GC, Toutouzas KG, Radin R, Chan L, Demetriades D. Nonoperative treatment of blunt injury to solid abdominal organs: a prospective study. Arch Surg. 2003; 138(8):844-51

7. Dent D, Alsabrook G, Erickson BA, Myers J, Wholey M, Stewart R et al. Blunt splenic injuries: high nonoperative management rate can be achieved with selective embolization. J Trauma. 2004; 56(5):1063-7.

8. Mclntyre LK, Schiff M, Jurkovich GJ. Failure of nonoperative management of splenic injuries: causes and consequences. Arch Surg. 2005; 140(6):563-8; discussion 568-9.

9. Fischer RP, Miller-Crotchett P, Reed RL. Gastrointestinal disruption: the hazard of nonoperative management in adults with blunt abdominal injury. J Trauma.1988; 28(10):1445-9.
10. Traub AC, Perry JF Jr. Injuries associated with splenic trauma. J Trauma. 1981; 21(10):840-7.

11. Federle MP, Courcoulas AP, Powel M, Ferris JV, Peitzman AB. Blunt splenic injury in adults: clinical and CT criteria for management, with emphasis on active extravasation. Radiology. 1998; 206(1):137-42

12. Pachter HL, Guth AA, Hofstetter SR, Spencer FC. Changing patterns in the management of splenic trauma: the impact of nonoperative management. Ann Surg. 1998; 227(5):708-17; discussion 717-9.

13. Peitzman AB, Heil B, Rivera L, Federle MB, Harbrecht BG, Clancy $K D$ et al. Blunt splenic injury in adults: Multi-institutional Study of the Eastern Association for the Surgery of Trauma. J Trauma. 2000; 49(2):177-89.

14. Shapiro MB, Nance ML, Schiller HJ, Hoff WS, Kauder DR, Schwab CW. Nonoperative management of solid abdominal organ injuries from blunt trauma: impact of neurologic impairment. Am Surg. 2001; 67(8):793-6.

15. Brasel KJ, Delisle CM, Olson CJ, Borgstrom DC. Splenic injury: trends in evaluation and management. J Trauma. 1998; 44(2):283-6.

16. Davis KA, Fabian TC, Croce MA, Gavant ML, Flick PA, Minard G et al. Improved success in nonoperative management of blunt splenic injuries: embolization of splenic artery pseudoaneurysms. J Trauma. 1998; 44(6):1008-15.

17. Ochsner MG, Knudson MM, Pachter L, Hoyt DB, Cogbill TH, McAuley CE et al. Significance of minimal or no intraperitoneal fluid visible on CT scan associated with blunt liver and splenic injuries: a multicenter analysis. J. Trauma. 2000; 49(3):505-10.

18. Myers JG, Dent DL, Stewart RM, Gray GA, Smith DS, Rhodes JE et al. Blunt splenic injuries: dedicated trauma surgeons can achieve a 
high rate of nonoperative success in patients of all ages. J. Trauma. 2000; 48(5):801-6.

19. Nix JA, Costanza M, Daley BJ, Powell MA, Enderson BL. Outcome of the current management of splenic injuries. J Trauma. 2001; 50(5):835-42.

20. Schurr MJ, Fabian TC, Gavant M, Croce MA, Kudsk KA, Minard G et al. Management of blunt splenic trauma: computed tomographic contrast blush predicts failure of nonoperative management. J Trauma. 1995; 39(3):507-13.

21. Godley CD, Warren RL, Sheridan RL, McCabe CJ. Nonoperative management of blunt splenic injury in adults: age over 55 years as a powerful indicator for failure. J Am Coll Surg. 1996; 183(2):133-9.

22. Konstantakos AK, Barnoski AL, Plaiser BR, Yowler CJ, Fallon WF Jr, Malangoni MA. Optimizing the management of blunt splenic injury in adults and children. Surgery. 1999;126(4):805-12; discussion 812-3.

23. Thaemert BC, Cogbill TH, Lambert PJ. Nonoperative management of splenic injury: are follow-up computed tomographic scans of any value? J Trauma. 1997; 43(5):748-51.

24. Uecker J, Pickett C, Dunn E. The role of follow-up radiographic studies in nonoperative management of spleen trauma. Am Surg. 2001; 67(1):22-5.

25. Fata P, Robinson L, Fakhry SM. A survey of EAST members practices in blunt splenic injury: a description of current trends and opportunities for improvement. J Trauma. 2005; 59(4):836-41; discussion 841-2.

26. Falimirski ME, Provost D. Nonsurgical management of solid abdominal organ injury in patients over 55 years of age. Am Surg. 2000; 66(7):631-5.

27. Krause KR, Howells GA, Bair HA, Glover JL, Madrazo BL, Wasvary $\mathrm{HJ}$, Bendick PJ. Nonoperative management of blunt splenic injury in adults 55 years and older: a twenty-year experience. Am Surg. 2000; 66(7)636-40.

28. Haan JM, Bochicchio GV, Kramer N, Scalea TM. Nonoperative management of blunt splenic injury: a 5-year experience. J Trauma. 2005; 58(3):492-8.

29. Pachter HL, Spencer FC, Hofstetter SR, Liang HG, Hoballah J, Copa GF. Experience with selective operative and nonoperative treatment of splenic injuries in 193 patients. Ann Surg. 1990; 211(5):583-9; discussion 589-91.

30. Peitzman AB, Harbrecht BG, Rivera L, Heil B; Eastern Association for the Surgery of Trauma Multiinstitutional Trials Workgroup. Failure of observation of blunt splenic injury in adults: variability in practice and adverse consequences. J Am Coll Surg. 2005; 201(2):179-87.

Recebido em 15/09/2008

Aceito para publicação em 20/11/2008

Conflito de interesse: nenhum

Fonte de financiamento: nenhuma

\section{Como citar esse artigo:}

Carvalho FH, Romeiro PCM, Collaço IA, Baretta GAP, Freitas ACT, Matias JEF. Fatores prognósticos relacionados à falha do tratamento não-operatório de lesões esplênicas no trauma abdominal fechado. Rev Col Bras Cir. [periódico na Internet] 2009; 36(2). Disponível em URL: http://www.scielo.br/rcbc

Endereço para correspondência:

Fábio Henrique de Carvalho

E-mail: fabiohc@uol.com.br 